\title{
Thermal-Structural Design and Optimization of Engine Exhaust-Washed Structures
}

\author{
Joshua D. Deaton ${ }^{1}$ and Ramana V. Grandhi ${ }^{2}$ \\ Wright State University, Dayton, OH, 45435
}

\begin{abstract}
Structures located aft of embedded engines on low observable aircraft, known as engine exhaust-washed structures (EEWS), are exposed to a combined loading environment that includes transient thermal effects in addition to conventional structural loading. Design in this environment is often complicated by non-intuitive, configuration-specific thermoelastic structural responses to elevated temperature and combined loading that result from design dependent thermal loads. In this work, the basic thermal-structural responses of EEWS components are explored to demonstrate the need for multidisciplinary optimization in EEWS design. A gradient-based thermal-structural optimization framework is developed to aid in the design of these components where thermal effects exhibit transient behavior. The framework involves properly updating both spatial and temporal design dependent thermal loads in structural optimization while managing transient thermoelastic responses as constraints. The framework is demonstrated on two structures that are subjected to combined thermal-structural loading where thermal effects are time varying.
\end{abstract}

\section{Nomenclature}

$A=$ cross section area

$A_{\text {conv }}=$ area factor for point convection

$B=$ buckling load factor

$B_{E}=$ Euler buckling number

$E=$ modulus of elasticity

$F=$ Hoffman composite failure index

$f=$ objective function

$h=$ convective film coefficient

$I=$ second moment of inertia

$L \quad=$ length

$P \quad=\quad$ axial load in element

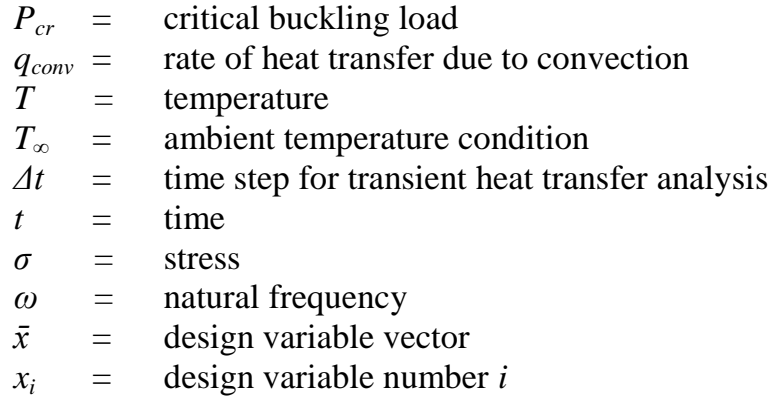

\section{Introduction}

The design of aerospace structures subjected to elevated temperature environments has been a critical area of research since the onset of supersonic flight in the early 1950's. As flight speeds increased, designers realized that the elevated temperatures resulting from high speed aerodynamic phenomena and their effects on aircraft structural performance would place a "thermal barrier" on supersonic flight. In response, a new area of research emerged known as thermal structures to help overcome this barrier with advances in aerospace materials and innovative structural designs. Basic considerations in the thermal structures field are related to two primary detrimental effects of elevated temperatures on aircraft structures. ${ }^{1}$ First, for most engineering materials, elevated temperatures decrease a structure's ability to carry load by reducing both elastic and failure strength properties. Second, elevated temperatures lead to both global and local thermal expansion of components that if restrained can lead to thermal stresses, thermal buckling behavior, and other thermoelastic responses. In conventional thermal structures design, the most common prescription for alleviating these phenomena is to simply accommodate the thermal expansion, which can altogether eliminate the most damaging thermoelastic effects and thermal stresses. ${ }^{2}$ One area where this prescription of allowing thermal expansion to prevent thermal stresses often cannot be applied is in the area of

\footnotetext{
${ }^{1}$ Graduate Research Assistant, Department of Mechanical and Materials Engineering, AIAA Student Member.

${ }^{2}$ Distinguished Professor, Department of Mechanical and Materials Engineering, AIAA Fellow.
} 
embedded engine integration on modern low observable aircraft. In this case, the ability to evade detection by an enemy supersedes many conventional structural design concerns.

\section{A. Embedded Engine Aircraft}

To meet the growing demands for improved mission capability, combat survivability, and versatility of aerospace systems, future military aircraft will continue to rely on low observable technology. A critical component of this technology is embedded engine integration, as seen on the B-2 Spirit stealth bomber platform and future low observable concepts. This configuration, in which engines are buried inside the aircraft, allows for a smooth outer mold line (OML), reduced exhaust noise, and cooler exhaust gases. These characteristics reduce the vehicle's observability by decreasing radar, acoustic, and infrared detectability. In addition, by utilizing a ducted exhaust system to pass exhaust gases to the rear of the aircraft, direct line of sight into hot engine components is prevented, denying the enemy a very vulnerable infrared target. ${ }^{3}$ This is demonstrated in Figure 1 with a simplified notional cross section of an embedded engine configuration with ducted exhaust. While such a configuration affords tremendous tactical capabilities, it also comes with increased structural design complexity. In a legacy aircraft, where engines are located either under the wings or in the aft fuselage, high temperature exhaust gases are expelled directly into the airstream, but in an embedded engine configuration the hot exhaust creates an extreme thermalstructural design environment as it is ducted to the rear of the aircraft. In this environment damaging effects of elevated temperatures including excessive deformations, thermal stresses, and thermal buckling may occur.

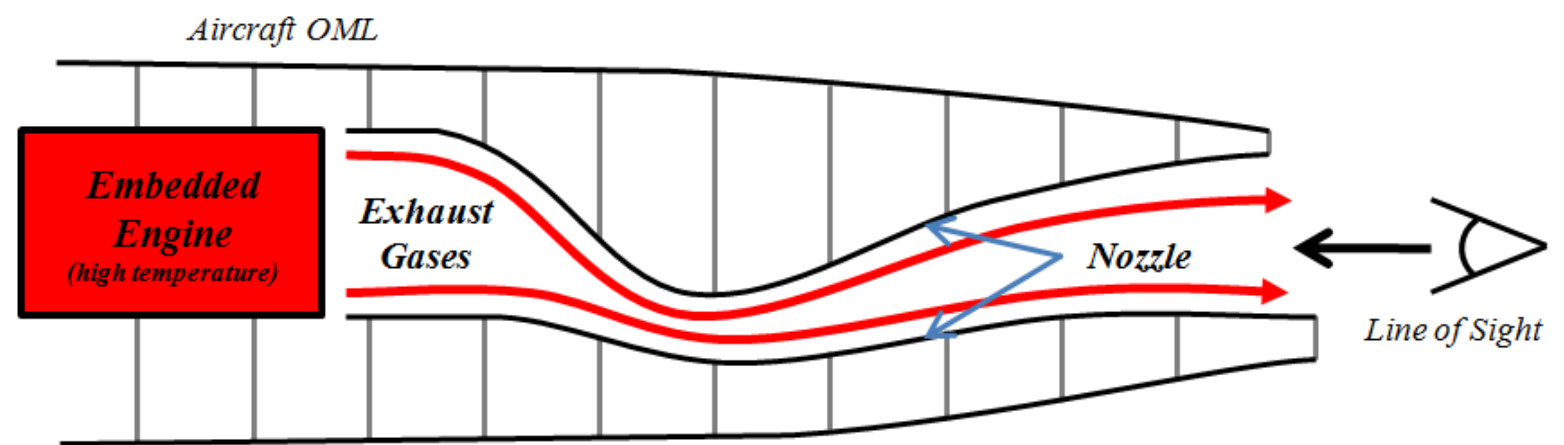

Figure 1. Notional cross section of an embedded engine configuration with a ducted engine exhaust nozzle.

\section{Engine Exhaust-Washed Structures}

The structural components located aft of the engines on an embedded engine aircraft that make up the ducted exhaust path are known as engine exhaust-washed structures (EEWS). ${ }^{4}$ In particular, exhaust-washed structures include the ducted nozzle itself, which is directly exposed to exhaust gases that may be in excess of $1000^{\circ} \mathrm{F}$, and the surrounding support structures to which it is attached. These components are subjected to a multidisciplinary loading environment where various effects of both thermal and structural loading produce a combined structural response. Thermal loads include transient elevated temperatures produced by the hot exhaust gases and aerodynamic effects that are driven largely by the mission profile of the aircraft. These effects produce both temporal and spatial temperature gradients that create varying amounts of thermal expansion at different times during a flight and at different locations throughout the structure. Additional structural loads experienced by engine exhaust-washed structures include pressure loading from the exhaust flow, air loads, potential mechanical loading reacted from adjoining airframe structures, and potential wide-band acoustic loading.

One of the primary challenges associated with the design of engine exhaust-washed structures is the dominance of low observability design criteria over conventional thermal-structural design concerns. In a typical configuration such as that shown in Figure 1, the geometric shape of the exhaust nozzle is fixed by propulsion system efficiency and low observability design criteria. In addition, all components must be smoothly integrated within the outer mold line of the aircraft. This creates a design space with strict geometric shape and fixivity constraints on structural components. Since all of these components are subjected to a spatially and temporally varying elevated temperature environment, the thermoelastic response of these structures will undoubtedly include restrained expansion in various areas of the overall structure. Thus, the major challenge for the designer is how to design this exhaust structure, the geometric shape parameters of which are typically fixed, and the surrounding substructure layout that must support it. Designers must accommodate thermal expansion effects to prevent excessive thermal stresses, which can lead to buckling and fatigue failures in the exhaust structures themselves, and also to prevent excessive thermal loads from 
being transferred to surrounding structures. This task is even more challenging because the thermal effects that typically drive the basic thermoelastic response of the structure are design dependent. This creates a complex multidisciplinary and often non-intuitive design space.

\section{A. Thermal-Structural Response}

The basic thermal-structural response of a conceptual engine exhaust-washed structure system was studied previously. ${ }^{5}$ In this work, sequential thermal-structural finite element analysis was used to determine the fundamental thermoelastic response of the structure when subjected to transient thermal effects and additional mechanical loading. The basic structural configuration with associated thermal and structural effects considered is given in Figure 2. Primary components include an inner nozzle through which high temperature exhaust is passed, the outer aircraft skins, and supporting substructure. Further details of the model, loading, and application of boundary conditions in the analysis are available in the referenced work.

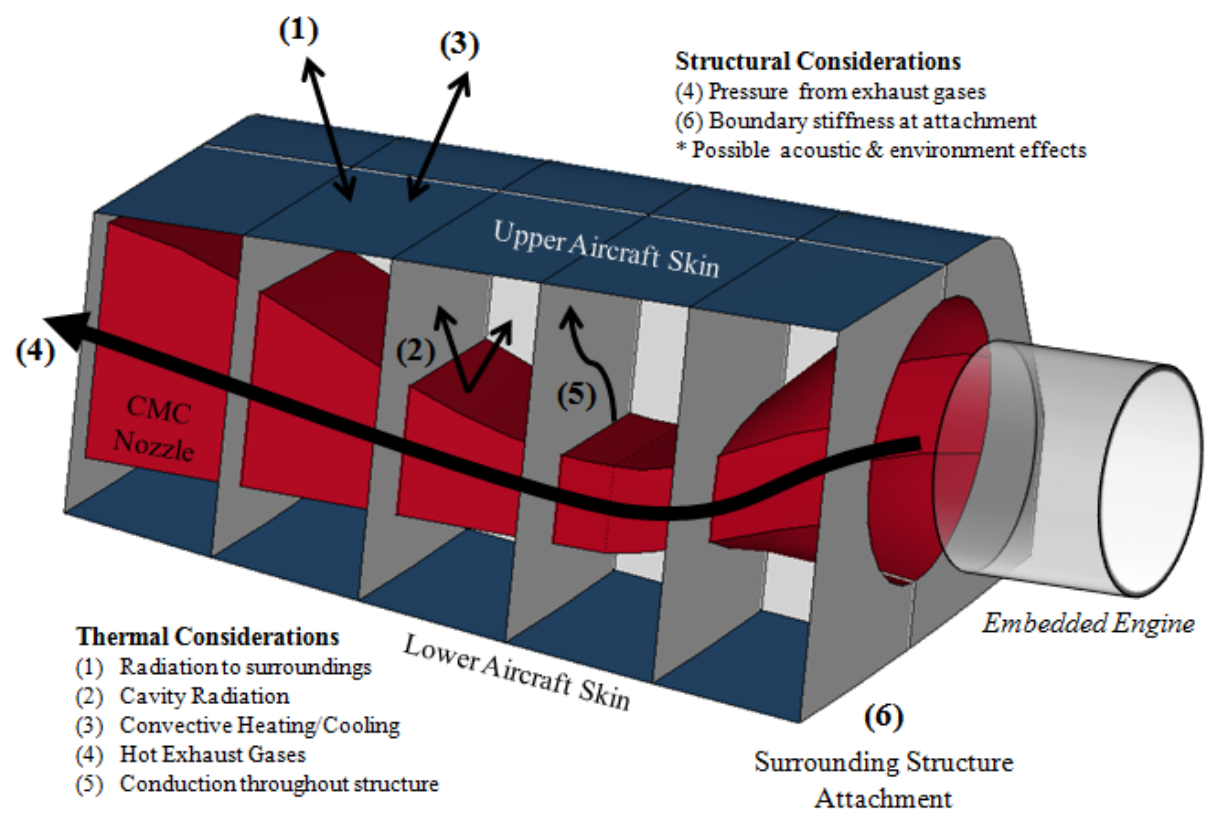

Figure 2. Engine exhaust-washed structure configuration with associated general loading and analysis considerations.

Figure 3(a) shows representative temperature results that were obtained during transient thermal analysis of the EEWS system. These include the time varying temperature of the nozzle structure (red areas in Figure 2), the average of all the nodal temperatures that make up the substructure (gray areas in Figure 2), and the average temperature of the outer skin nodes (blue areas in Figure 2) at each time step. We note that throughout the transient analysis, the nozzle portion of the structure is typically at much higher temperatures than the surrounding structures. The temperatures of surrounding substructure and outer aircraft skins increase as heat is conducted from the hot nozzle throughout the rest of the structure, with some amount of time lag due to the transient thermal effects.

The resulting temperature fields that were obtained at each discrete time step in the analysis were then mapped to a structural model to determine the thermoelastic response of the system. Additional considerations in the structural model included a pressure load exerted normal to the inside of the nozzle surface and elastic boundary conditions. The maximum von Mises stress measured in the nozzle structure and the maximum equivalent strain within the supports at each time step are plotted in Figure 3(b). In this system, the nozzle structure is made of a CMC material that will likely fail due to excessive stresses. The substructure is made of composite materials, so equivalent strain is selected to gage their overall response to the loading. We note that values of each response tend to follow the overall magnitude of temperature of the structure, with the maximum nozzle stress and maximum substructure strain occurring when the nozzle is at its maximum temperature. This indicates that the fundamental response of the system is driven by the thermal expansion of the much hotter inner nozzle being restrained by the cooler outer substructure and aircraft skins. An interesting note is that maximum values do not occur simply when the overall temperature of the structure is at a maximum, but rather during times with the greatest spatial temperature difference between the nozzle and the surrounding structure. This is evident in the stress and strain results between roughly 720 
seconds and 900 seconds in Figure 3(b). We note a decrease in both the nozzle stress and substructure strain as the average temperature of the substructure increases during this time period, which can be observed in Figure 3(a). This temperature increase creates more expansion in the substructure, which relaxes the restraints on the greater nozzle expansion. This basic behavior is characteristic of built up exhaust structure systems. That is, critical structural responses do not occur simply at overall maximum temperatures, but at greatest spatial difference in temperature between different structural components.

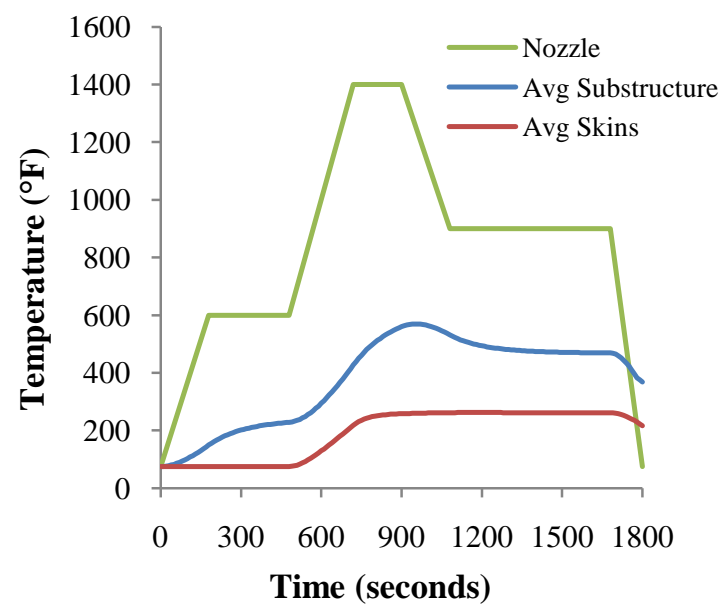

(a) Thermal analysis results

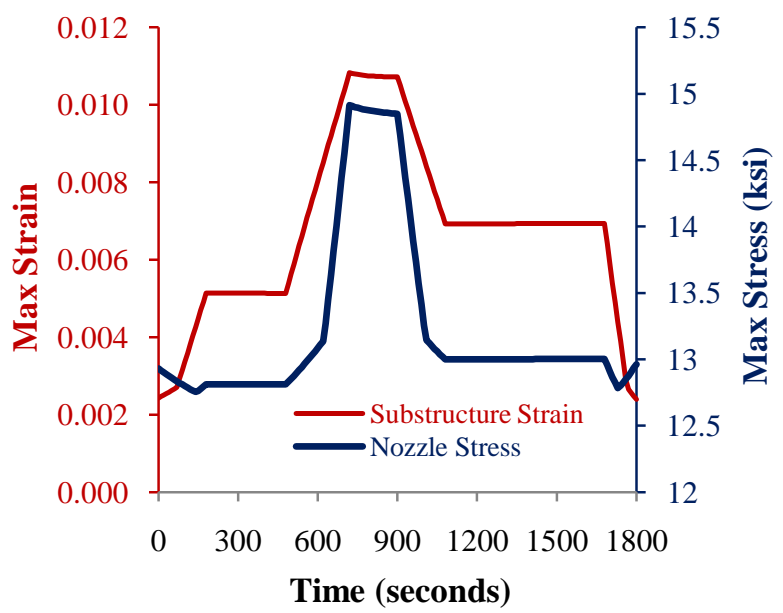

(b) Structural analysis results

Figure 3. Summarized results at each time step of thermal-structural analysis of the engine exhaustwashed structure.

\section{B. Parametric Analysis}

To investigate the effects of dimensional variation on the basic EEWS structural response, a parametric analysis was conducted on the same conceptual EEWS structure as the previously referenced work. The thickness of the nozzle portion of the structure and the thickness the transverse support component of the substructure were varied as a ratio of the baseline values used in the previous work. The thicknesses of other components were not varied because primary responses are most sensitive to variability in the nozzle and transverse supports. These thickness components are highlighted in Figure 4.
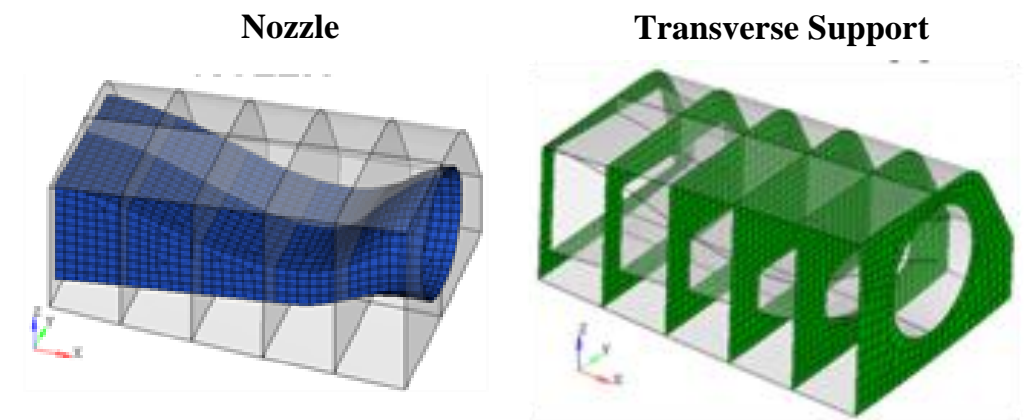

Figure 4. Component thickness parameters varied in the EEWS parametric analysis.

Figure 5(a) shows the resulting change in the maximum von Mises stress in the nozzle, while Figure 5(b) shows the change in the maximum equivalent strain in the substructure. The variation in each response is due to the design dependence of thermoelastic effects in the system. As we increase the thickness of one component, we observe an increase in the recorded structural response of the other. For example, increasing the thickness of the nozzle structure reduces its own stress (as we might expect), but causes an increase in the substructure strain. This occurs because by increasing the nozzle thickness, we have added more material that also undergoes thermal expansion due to the elevated temperatures. This creates more loading that must be carried by the substructure, which increases the 
strain as observed. Likewise, increasing the thickness of the transverse supports decreases the equivalent strain seen in the substructure, but causes a slight increase in the von Mises stress in the nozzle. This occurs because by increasing the thickness of the substructure, we have effectively stiffened the boundaries that are restraining the expansion of the nozzle, which leads to an increase in nozzle stress. Thus a trade-space in the sizing of exhaustwashed structures is observed.

(a) Nozzle Stress (psi)

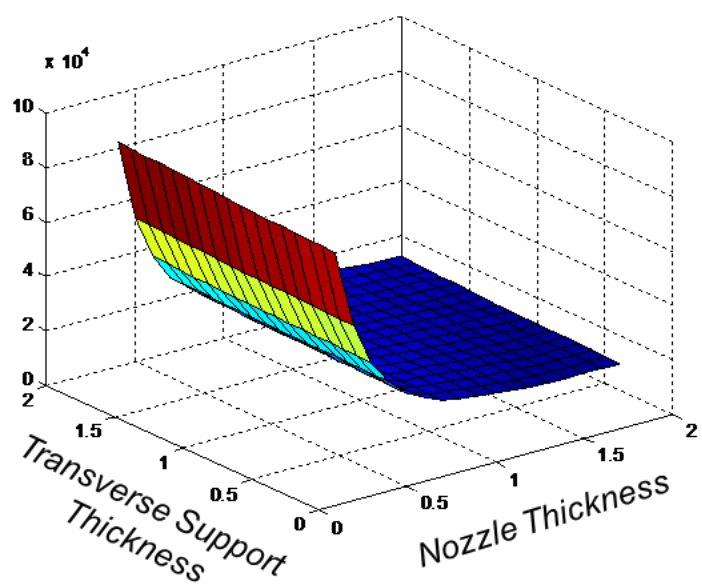

(b) Substructure Strain

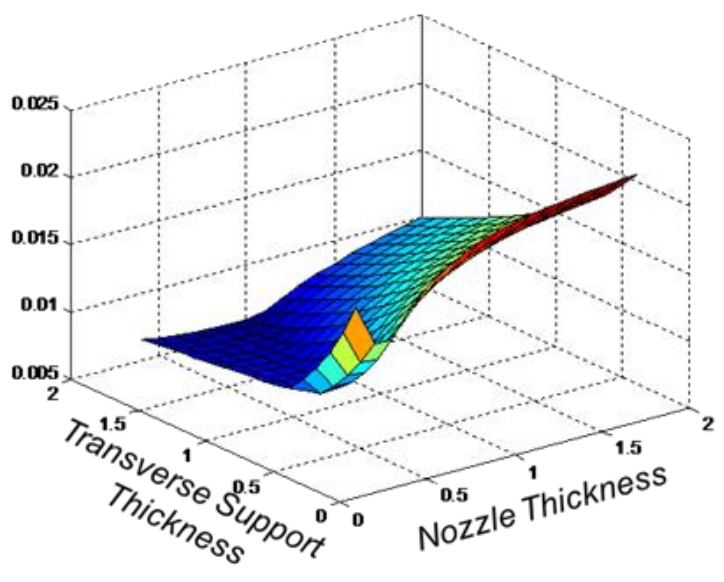

Figure 5. EEWS parametric analysis results. (a) Nozzle von Mises stress variation. (b) Substructure equivalent strain variation.

The parametric analysis results are similar to work in the literature related to the design of structures with restrained thermal expansion. ${ }^{6}$ Using an isotropic beam strip model subjected to a uniform temperature increase, Haney et al demonstrated that depending on the stiffness of the beam and its boundaries, increasing the thickness of the beam (a common prescription for both preventing thermal buckling or decreasing stress in a mechanically loaded component) may actually cause an increase in the stress in the beam. This resulted from the increased material that also undergoes thermal expansion. Thus, thermal buckling of the beam may be suppressed, but increased stresses could still lead to failure. An increase in the reaction loads at the beam's boundary is also noted. In an actual system, this means that greater forces would be applied to adjoining structures. The same work also indicates a heavy dependence of other responses such as thermal buckling on component boundary stiffness. In summary, caution is advised before applying conventional room temperature mechanical design practices when working in a thermal environment and demonstrates the basic non-intuitiveness of the exhaust-washed structure design space.

Due to the need to manage a complex thermal-structural response, design dependent loading, multiple opposing structural responses, and an overall non-intuitive design space we apply multidisciplinary optimization methods to the design of engine exhaust-washed structures.

\section{Thermal-Structural Optimization}

Design for thermal structures and thermal-structural optimization is typically carried out in a multidisciplinary, coupled manner where the resulting temperature fields from a heat transfer analysis are mapped onto a structural model where additional mechanical loading and mechanical boundary conditions are applied. ${ }^{7,8}$ Inside the structural analysis, the nodal temperature information is used to compute thermoelastic response of the structure. It should be noted that this thermoelastic response is design dependent at two levels. First, as previously discussed, a change in a particular design variable in a basic sizing optimization may change the thermoelastic response by adding or removing structural material that undergoes thermal expansion. Perhaps more important, a change in design variables will also modify the temperature field that results from heat transfer analysis. This could result in a large change in the overall thermoelastic response of the structure depending on the sensitivity of the temperature fields to the design variability. This multi-level design dependency is accounted for by simply including the heat transfer analysis inside the design loop to compute new temperature fields at each design iteration. This capability exists in various commercial analysis and optimization packages including Nastran and GENESIS, but only for steady-state heat transfer analyses. ${ }^{9,10}$ This prevents the direct application of these tools to thermal-structural optimization 
problems where the thermal effects exhibit transient time varying behavior as commonly found in engine exhaustwashed structures.

For a large number of thermal-structural optimization problems, this methodology may be adequate because the worst-case thermoelastic response occurs when the overall magnitude of the temperature field throughout is at its highest. This commonly occurs when the structure has reached steady-state. As a result, a common design practice is to select the boundary conditions and thermal loading conditions that will result in the maximum temperature field during heat transfer analysis. However, this practice is not applicable in the design of engine exhaust-washed structures. In these components, as earlier identified, the critical thermoelastic responses tend to occur when the inner regions of the structure are at a much higher temperature than the outer regions. Steady-state results allow the substructure regions of the built up structure to come up to temperature. Thus, steady state results are unable to capture the temporally varying critical thermoelastic responses. In some cases this may result in ineffective designs when subjected the actual transient loading conditions, while in others it may result in overly conservative designs.

To properly account for the transient nature of the EEWS design problem, a transient optimization formulation is required that can capture both the spatial and temporal design dependency of the thermal-structural response. As in thermal-structural optimization involving steady-state heat transfer analysis, accounting for the effect of design variability in the resulting temperature fields requires updated transient heat transfer results. The primary challenge with this is the transient thermal analysis is typically several orders of magnitude more computationally expensive than basic steady-state thermal analysis. Thus, a reanalysis of transient temperature fields at each structural design iteration is infeasible in many full-scale design problems. In addition, the consideration of transient thermal loading means that the constraints in the system will be transient as well. This can result in a large number of constraints for comparatively few distinct responses (stresses or displacements at a particular location for example) because the constraints must be enforced not only at various locations throughout the structure, but also at each point in time. ${ }^{11}$ The next section discusses a transient thermal-structural optimization framework that has been developed to overcome these challenges to solve larger scale thermal-structural optimization problems.

\section{A. Transient Optimization Framework}

A basic framework for gradient-based, transient optimization of thermal-structural problems, where thermal effects are transient in nature, has been developed. The program flow of the framework is shown in Figure 6. The overall operation is based on a structural optimization loop inside an outer loop. Transient heat transfer analysis to obtain grid point temperature fields is performed in MD Nastran using a SOL400 time stepping scheme. ${ }^{9}$ The resulting temperature fields (one per output time step) are used in the construction of a GENESIS input file to perform structural analysis and optimization. ${ }^{10}$ The GENESIS input consists of one static loadcase for each time step temperature field. Combined mechanical loads are also included in these loadcases to obtain the thermoelastic structural response at each discrete point in time. Extra loadcases per each time step can be added to calculate other structural responses that may depend on the time varying thermal-mechanical loads, for example linear buckling. Relevant constraints are placed on each of these loadcases to ensure that during optimization, no response violates a constraint at any point in time for the given temperature field set. In addition, other analyses are considered such as a modal analysis to enforce a minimum natural frequency for a structure. Depending on design problem size, GENESIS's built in DOT or BIGDOT optimizer is used for structural optimization.

Once GENESIS has converged to an optimum for the specified loadcases, the process is looped back to another Nastran transient heat transfer analysis. This provides updated thermal loading to a new GENESIS structural optimization. This process is repeated until convergence of the temperatures at each grid and each time step between successive outer loops is reached. This indicates that between the last two structural optimization runs, design variable changes were small enough that they did not significantly impact the transient heat transfer throughout the structure. Thus, the final structural optimization is performed on the proper transient temperature fields. While this outer loop heat transfer analysis is not efficient from strictly an optimization perspective because sensitivity analysis does not see the heat transfer design dependency, as previously stated, reanalysis of a transient heat transfer solution for a problem of marginal size is typically not possible due to computational and time requirements. Thus, while this outer loop reanalysis process may require in total more "structural" optimization iterations, these computations are relatively inexpensive compared to the cost of executing a transient heat transfer analysis with each structural design cycle as is possible when using steady-state heat transfer analysis.

Currently, there is one minor limitation to this framework. While MD Nastran SOL400 is able to accommodate temperature dependent properties in transient heat transfer analysis, GENESIS is unable to use temperature dependent mechanical properties in structural analysis. The error introduced by this limitation is currently minimized by using mechanical properties at the anticipated elevated temperatures. This accounts for the loss in stiffness and failure strength that typically occur due to elevated temperatures. 


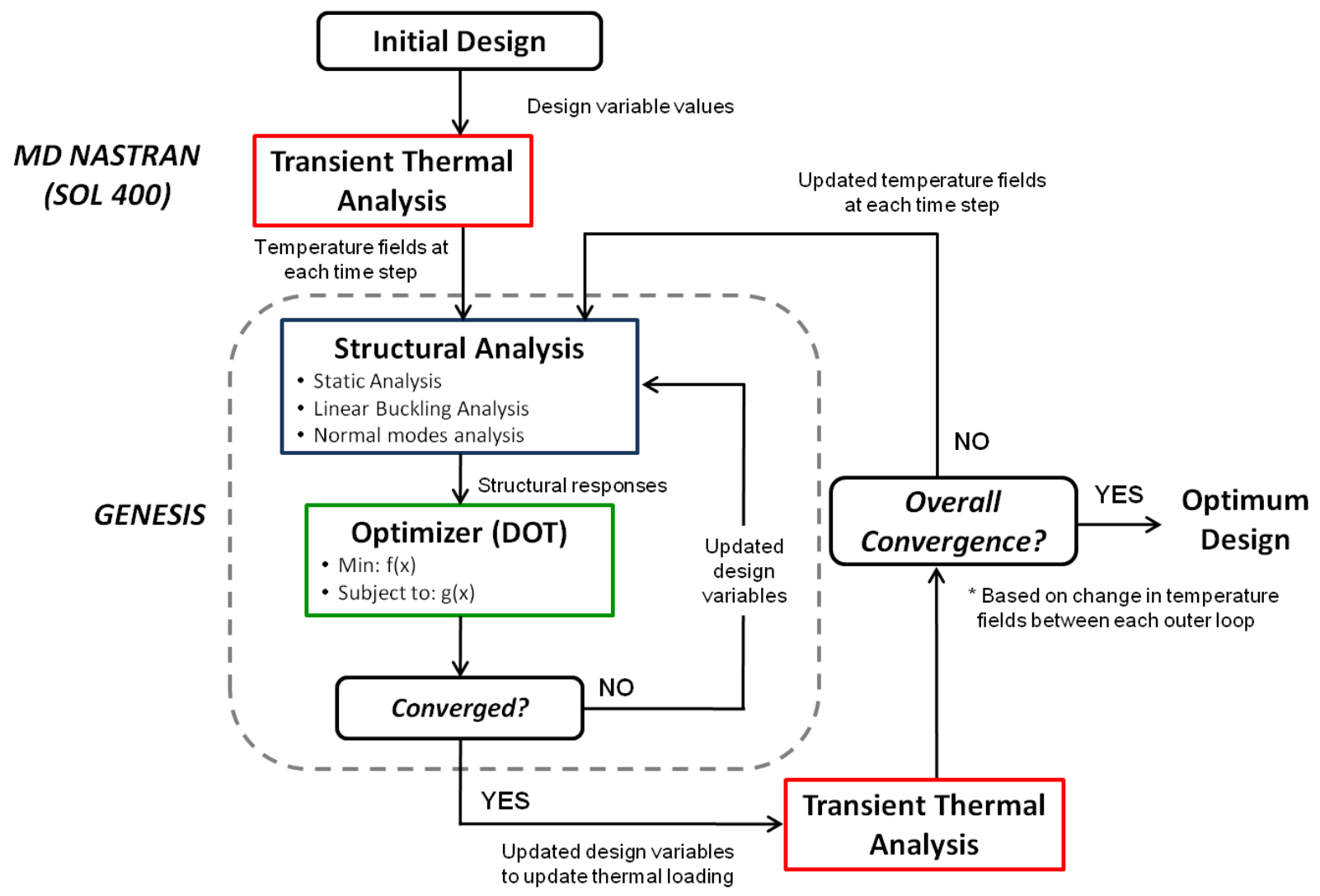

Figure 6. Transient thermal-structural optimization framework using the commercial packages MD Nastran and GENESIS.

\section{Demonstration}

In this section we demonstrate the developed transient thermal-structural optimization framework on two different structures that are subjected to combined thermal-structural loading. We focus on loading scenarios where thermal effects on the structures are transient, which creates temporal temperature variations and temporal thermoelastic responses, as previously identified on engine exhaust-washed structures.

\section{A. Three Bar Truss Structure}

The first demonstration example is a simple three bar truss structure that is subjected to combined thermal and mechanical loading. In this example, we explore the application of the optimization framework to the problem and the resulting design dependency of the temperature field resulting from thermal analysis throughout.

The three bar truss model and associated combined thermal-structural loading is given in Figure 7. The model consists of four nodes connected by 3 rod elements. It is assumed that the rods are of solid circular cross section. Thermal and mechanical properties of the rods are taken as Titanium 6-4 alloy at $700^{\circ} \mathrm{F}$ and are given in Table 3 in the Appendix section. ${ }^{12}$

In transient thermal analysis the temperature of node (1) is a time varying controlled temperature as given in Figure 8. Convective heat losses from the structure at nodes (2) and (3) to an ambient condition are modeled as point convection given by Equation (1).

$$
q_{\text {conv }}=h A_{\text {conv }}\left(T-T_{\infty}\right)
$$

The area factor, $A_{\text {conv }}$, for each node is assumed to be 1.0 and the convective film coefficient, $h$, at nodes (2) and

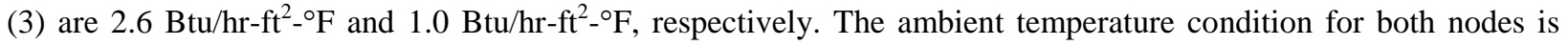


taken as a constant $T_{\infty}=75^{\circ} \mathrm{F}$ throughout the transient analysis. Thus, the overall transient thermal response can be summarized as heat loss from high temperature at node (1) through conduction at node (4) to nodes (2) and (3) where it is convected to the ambient condition.

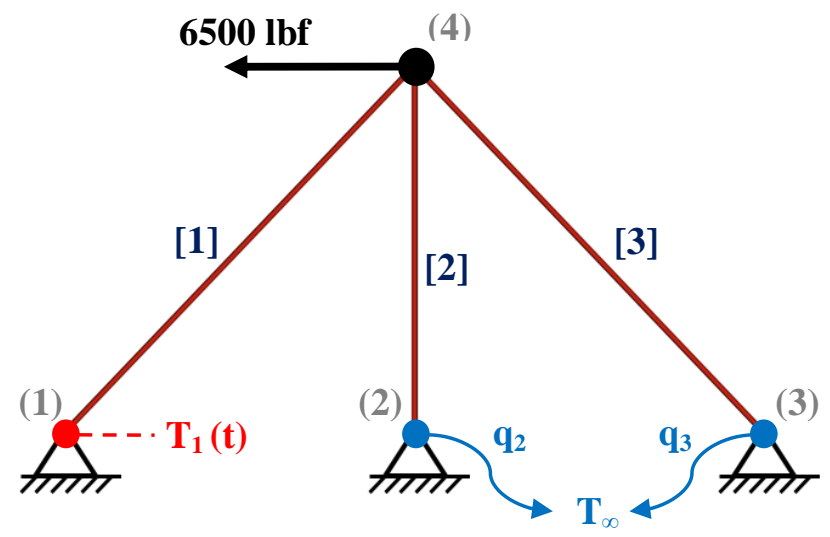

Figure 7. Three bar truss structure with combined thermal and mechanical loads.

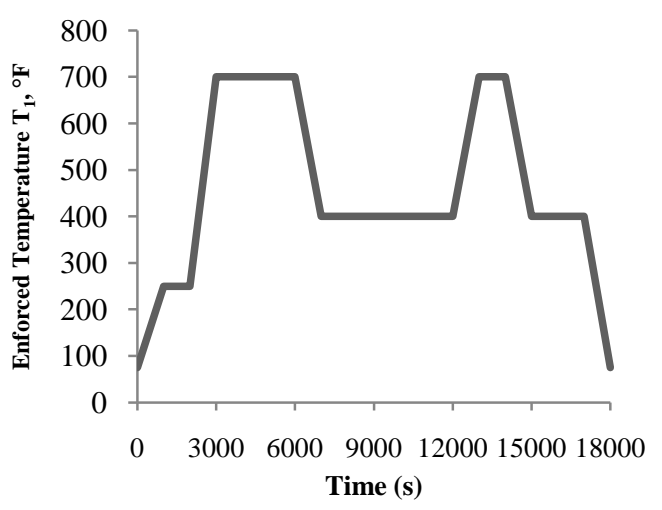

Figure 8. Time varying enforced temperature of node (1) in the three bar truss model.

In the transient thermal analysis, the time step, $\Delta t$, is taken as 1 second during simulation, with output temperature fields created in 10 second intervals. Thus, for the 18,000 second transient heat transfer analysis, a total of 1801 discrete temperature fields are passed to the structural analysis/optimization.

Mechanical boundary conditions are modeled as pinned restraints at nodes (1), (2), and (3). A $6500 \mathrm{lb}$ mechanical load is applied at node (4) as well as a concentrated mass of $10 \mathrm{lb}$. Structural responses of interest include the first natural frequency of the structure, axial stresses in each bar, and the Euler buckling conditions for each rod. To prevent buckling, the compressive force in each rod element must remain above the Euler buckling load as given by Equation (2). ${ }^{13}$ Thus, for a circular cross section $\left(I=A^{2} / 4 \pi\right)$, we can arrange Equation (2) into a constraint value as given in Equation (3).

$$
\begin{gathered}
P \geq P_{c r}=\frac{\pi^{2} E I}{L^{2}} \\
B_{E}=\frac{P L^{2}}{A^{2}} \geq-\frac{\pi E}{4}
\end{gathered}
$$

Design variables in the problem consists of the three bar areas $\left(\bar{x}=\left[A_{1} A_{2} A_{3}\right]\right)$. Overall convergence is based on a maximum temperature change at any node at any point in time of $1{ }^{\circ} \mathrm{F}$ between sequential outer loops. To clarify, convergence is reached when the difference between transient heat transfer results at any node and at any time step is no larger than $1^{\circ} \mathrm{F}$.

The optimization problem is given as follows:

Minimize: $f(\bar{x})=$ structural weight (not including concentrated mass)

Subject to: Axial stress: $\quad-25.0 \leq \sigma(\bar{x}, t) \leq 25.0 \mathrm{ksi}$

Euler Buckling: $\quad B_{E}(\bar{x}, t) \geq-9.719$ Msi

Natural Frequency: $\quad \omega_{1}(\bar{x}) \geq 20 \mathrm{~Hz}$

Design variables: $\quad 0.1 \leq \bar{x} \leq 2.0 \mathrm{in}^{2}$

The results of the thermal-structural optimization are summarized in Table 1. The initial design variable vector is $\bar{x}=\left[\begin{array}{lll}1.0 & 1.0 & 1.0\end{array}\right]$, which corresponds to a structural weight of $14.7 \mathrm{lb}$. In the first outer loop, a given set of transient nodal temperatures is obtained and is used in the first structural optimization run. We see that this is initially an infeasible design, but after the first structural optimization (requiring 5 design iterations) all constraints are satisfied for the given load set. Loop 2 begins with the optimum design that resulted from Loop 1, and a new transient heat transfer analysis is conducted to obtain new temperature data. We note that temperature values show a significant change resulting from the design variable changes in Loop 1. At the beginning of structural optimization with the 
new load set, we see that both the buckling and stress constraints are violated at some point in time. After four structural design iterations in Loop 2, we have converged to a new optimum for the given transient load set, and Loop 3 again begins with an updated heat transfer analysis. This process repeats once more until total convergence is reached between Loop 3 and Loop 4. At this time, design variable changes in the structural optimization of Loop 3 did not produce enough variability to significantly change the resulting temperatures. Thus total convergence is reached. The resulting design is $\bar{x}=\left[\begin{array}{l}0.9530 .1730 .466\end{array}\right]$ with a structural weight of 8.37 pounds. Buckling and stress constraints are active in the final design at some point in time. The frequency constraint is also active.

The largest design drivers appear to be tensile stresses in element 2, buckling in element 1 , and the minimum frequency constraint. An optimum design was ultimately reached by reducing the area of element 2 as much as possible (inside the axial stress constraint) and element 3 (inside the frequency constraint), which ultimately allowed more expansion of element 1 (which was always at higher temperature). By allowing more expansion of this element, less compressive force is exerted which makes it less likely to buckle. In addition, re-evaluation of the optimization problem with various initial design variable values within the bounds converges to the same optimum solution.

Table 1. Thermal-structural optimization results for three bar truss problem.

\begin{tabular}{|c|c|c|c|c|c|c|c|c|}
\hline & \multicolumn{2}{|c|}{ Loop 1} & \multicolumn{2}{|c|}{ Loop 2} & \multicolumn{2}{|c|}{ Loop 3} & \multicolumn{2}{|c|}{ Loop 4} \\
\hline & \multicolumn{2}{|c|}{5 Design Iterations } & \multicolumn{2}{|c|}{4 Design Iterations } & \multicolumn{2}{|c|}{2 Design Iterations } & \multicolumn{2}{|c|}{0 Design Iteration } \\
\hline & Initial & Optimum & Initial & Optimum & Initial & Optimum & Initial & Optimum \\
\hline$f(\bar{x})(\mathrm{lb})$ & 14.70 & 7.78 & 7.78 & 8.45 & 8.45 & 8.37 & 8.37 & \multirow{7}{*}{ 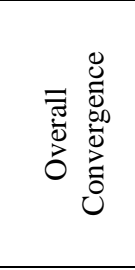 } \\
\hline$A_{l}\left(\mathrm{in}^{2}\right)$ & 1.00 & 0.866 & 0.866 & 0.961 & 0.961 & 0.953 & 0.953 & \\
\hline$A_{2}\left(\mathrm{in}^{2}\right)$ & 1.00 & 0.100 & 0.100 & 0.179 & 0.179 & 0.173 & 0.173 & \\
\hline$A_{3}\left(\mathrm{in}^{2}\right)$ & 1.00 & 0.496 & 0.496 & 0.468 & 0.468 & 0.466 & 0.466 & \\
\hline$*$ Euler Buckling, $B_{E}(\mathrm{Msi})$ & -13.85 & -9.668 & -10.08 & -9.664 & -9.618 & -9.681 & -9.685 & \\
\hline$*$ Axial Stress, $\sigma(\mathrm{ksi})$ & 10.50 & 23.94 & 27.69 & 24.96 & 24.67 & 24.86 & 24.88 & \\
\hline Natural Frequency, $\omega_{l}(\mathrm{~Hz})$ & 24.90 & 20.15 & 20.15 & 20.16 & 20.16 & 20.10 & 20.10 & \\
\hline$* * \operatorname{Max} \Delta T\left({ }^{\circ} \mathrm{F}\right)$ & & \multicolumn{2}{|c|}{80.89} & & & \multicolumn{2}{|c|}{0.96} & \\
\hline & \multicolumn{8}{|c|}{ * maximum value at any point in time } \\
\hline & \multicolumn{8}{|c|}{ ** maximum absolute change at any grid point at any time between sequential outer loops } \\
\hline & & \multicolumn{7}{|c|}{ violated constraint value } \\
\hline
\end{tabular}

\section{B. Composite Plate Structure}

A second demonstration example consists of a beam-like plate structure that represents part of a simplified composite EEWS subjected to combined thermal-structural loading. Again, thermal effects are transient in nature. In this example, we focus on the application of the process to a larger scale structure with similar loading to an actual EEWS system.

The finite element model of the structure with associated thermal-structural loading and boundary conditions is given in Figure 9. Five designable components are also labeled in the figure. The overall dimensions of the model are $96 \times 20 \times 24$ in. The upper red colored portion of the model represents the surface over which hot exhaust gases pass and is made of a CMC material to withstand the high temperatures. The rest of the structure, which represents adjoining aircraft substructure, is modeled with a sandwich composite material comprised of a titanium honeycomb core and graphite-bismaleimide (Gr/BMI) facesheets, which has good performance at mildly elevated temperatures. The facesheets are considered symmetric over the core and each have a stacking sequence of $[ \pm 45 / 0 / 90]_{s}$. The fundamental thermal and mechanical properties of the composite materials used in the analyses are given in Table 4 in the Appendix. Properties are approximated based on commercially available materials. ${ }^{14-16}$ Since documented thermal properties for the exact facesheet material are unavailable in the literature, they are approximated using available graphite-epoxy properties, which are expected to be very similar. ${ }^{17}$ In addition, thermal properties of the titanium honeycomb core material are intuitively approximated based on properties of a similar aluminum honeycomb material system. ${ }^{18}$ While it is acknowledged that exact and validated properties should be used in an actual design problem, these approximations are sufficient within the context of this example problem. 


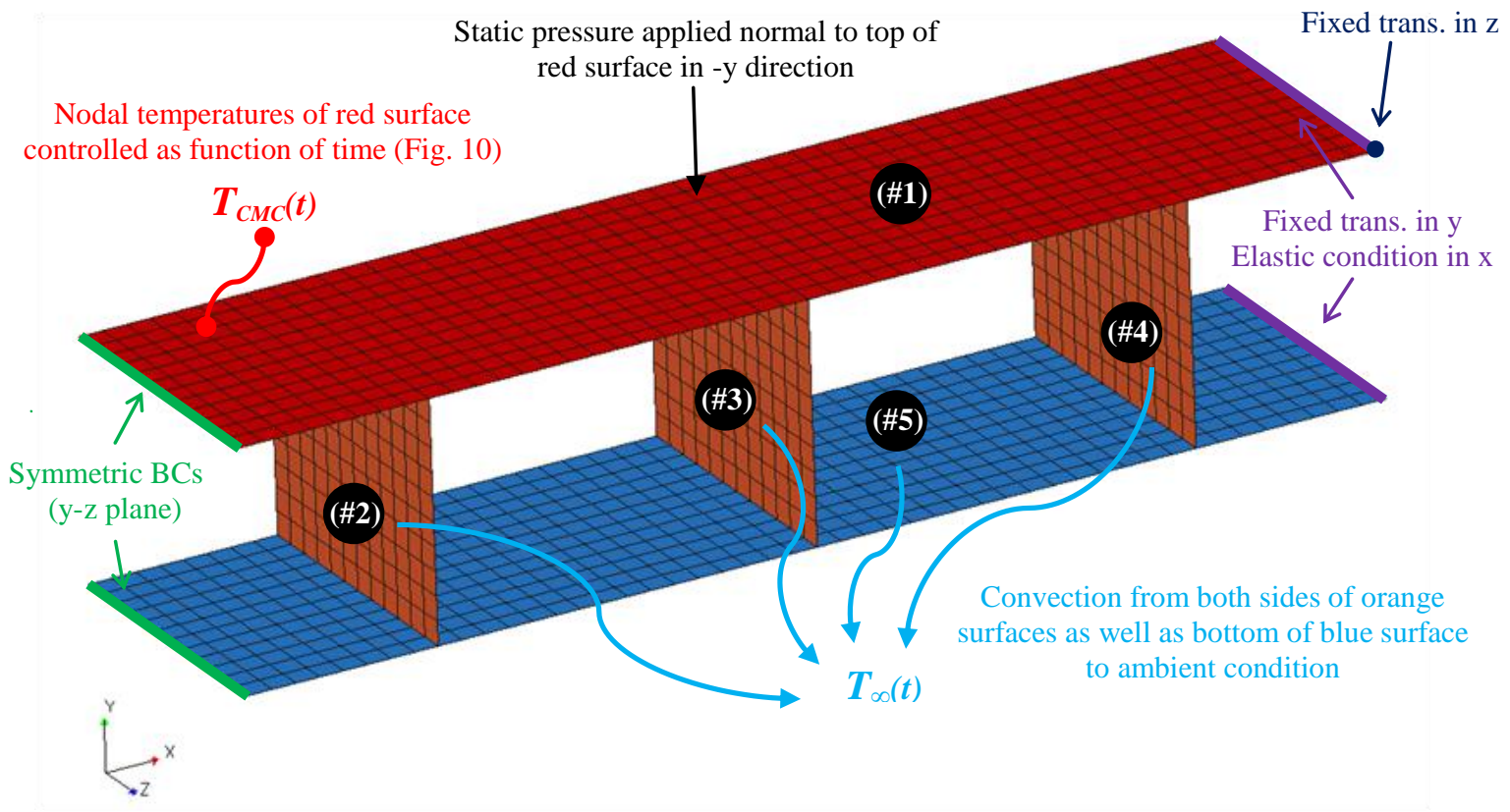

Figure 9. Finite element model for the second demonstration problem with associated thermal-structural loading and boundary conditions.

Thermal effects on the structure consist of a time varying temperature boundary condition on the CMC surface (\#1) to represent transient exhaust heating consistent with changes during component operation and convection boundary conditions to an ambient point on other components to represent active cooling/ventilation and aerodynamic cooling. The convection boundary conditions have film coefficients of $10.0 \mathrm{Btu} / \mathrm{ft}^{2}-\mathrm{hr}-{ }^{\circ} \mathrm{F}$ and 20.0 $\mathrm{Btu} / \mathrm{ft}^{2}-\mathrm{hr}-{ }^{\circ} \mathrm{F}$ for components (\#2) to (\#4) and (\#5), respectively. In both cases, the ambient temperature condition is assumed to be time varying. The time dependency of the CMC temperature and ambient temperature condition are given in Figure 10. In addition, a 0.5 psi pressure is applied normal to the CMC surface (\#1). In an actual EEWS simulation, this mechanical load would be time varying according to the mission profile and would typically be of greater magnitude. It is taken as constant and reduced magnitude to place emphasis on transient thermal effects in this demonstration. Structural boundary conditions consist of symmetry over the y-z plane at the left end of the structure. On the right end, translation at each grid point is fixed in y direction, and elastic spring elements provide stiffness against $\mathrm{x}$-translation. This represents the elastic restraint that would resist thermal expansion of the structure from the surrounding airframe on an actual engine exhaust-washed structure configuration.

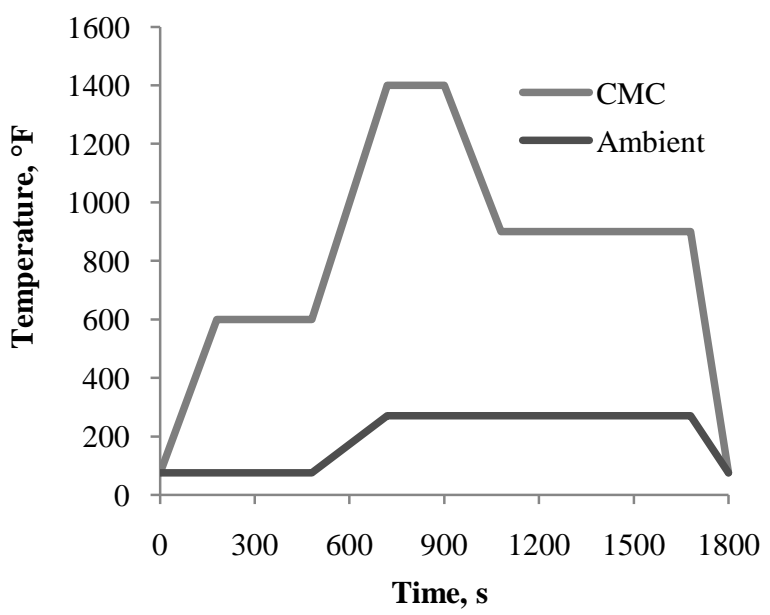

Figure 10. Time varying enforced temperature of CMC and ambient temperatures.

10

American Institute of Aeronautics and Astronautics 
In transient heat transfer analysis, a time step of 1 second is used, with temperature fields created every 10 seconds. Throughout the 1800 s simulation, this results in 181 discrete grid temperature sets that are used in each inner structural optimization.

In engine exhaust-washed structure designs, thermal buckling is a fundamental concern in addition to basic material failure. Thus, design responses include the Hoffman failure criteria as well as the buckling load factor obtained from a linear buckling analysis. For this problem 181 static loadcases are required in addition to 181 buckling loadcases to approximately capture the combined thermal-structural effects throughout each point in time.

The basic optimization problem is to determine the overall thickness of the CMC component (\#1), the honeycomb core thickness for each of the sandwich components (\#2) through (\#5), and the thickness of each ply orientation $(0 / 90 / \pm 45)$ of the composite facesheets. It is assumed all components have the same facesheet properties and the \pm 45 ply thicknesses are linked throughout. This results in a design problem with a total of eight individual design variables. The thickness of the honeycomb core in each component has a large effect on the buckling response, while sizing the thickness of each ply orientation helps to determine how many plies in each direction are required to optimally satisfy design requirements. Placing a lower bound on the thickness of each ply orientation equal to the thickness of one ply ( 0.005 inches) ensures that at least some plies in each primary direction exist to support possible unexpected off-direction loading. This is consistent with standard composite material design practices. ${ }^{19,20}$

The optimization problem is given as:

Minimize: $f(\bar{x})=$ structural weight

Subject to: $\quad$ Hoffman Failure Criteria: $F(\bar{x}, t) \leq 0.9$

Buckling Load Factor: $B(\bar{x}, t) \geq 1.5$

Design variables: CMC (\#1) Thickness: $\quad 0.2 \leq x_{1} \leq 2.0$ in

$0^{\circ}$ Ply Thicknesses: $\quad 0.005 \leq x_{2} \leq 0.07$ in

$90^{\circ}$ Ply Thicknesses: $\quad 0.005 \leq x_{3} \leq 0.07$ in

$\pm 45^{\circ}$ Ply Thicknesses: $0.005 \leq x_{4} \leq 0.07$ in

(\#2) Core Thickness: $\quad 0.25 \leq x_{5} \leq 0.75$ in

(\#3) Core Thickness: $\quad 0.25 \leq x_{6} \leq 0.75$ in

(\#4) Core Thickness: $\quad 0.25 \leq x_{7} \leq 0.75$ in

(\#5) Core Thickness: $\quad 0.25 \leq x_{8} \leq 0.75$ in

A maximum of 15 inner structural optimization iterations is also specified for each outer loop. Overall convergence is based on a maximum absolute temperature change at any node at any time-step of $10.0^{\circ} \mathrm{F}$. The results of the thermal-structural optimization are given in Table 2.

Table 2. Thermal-structural optimization results for simplified composite plate structure problem.

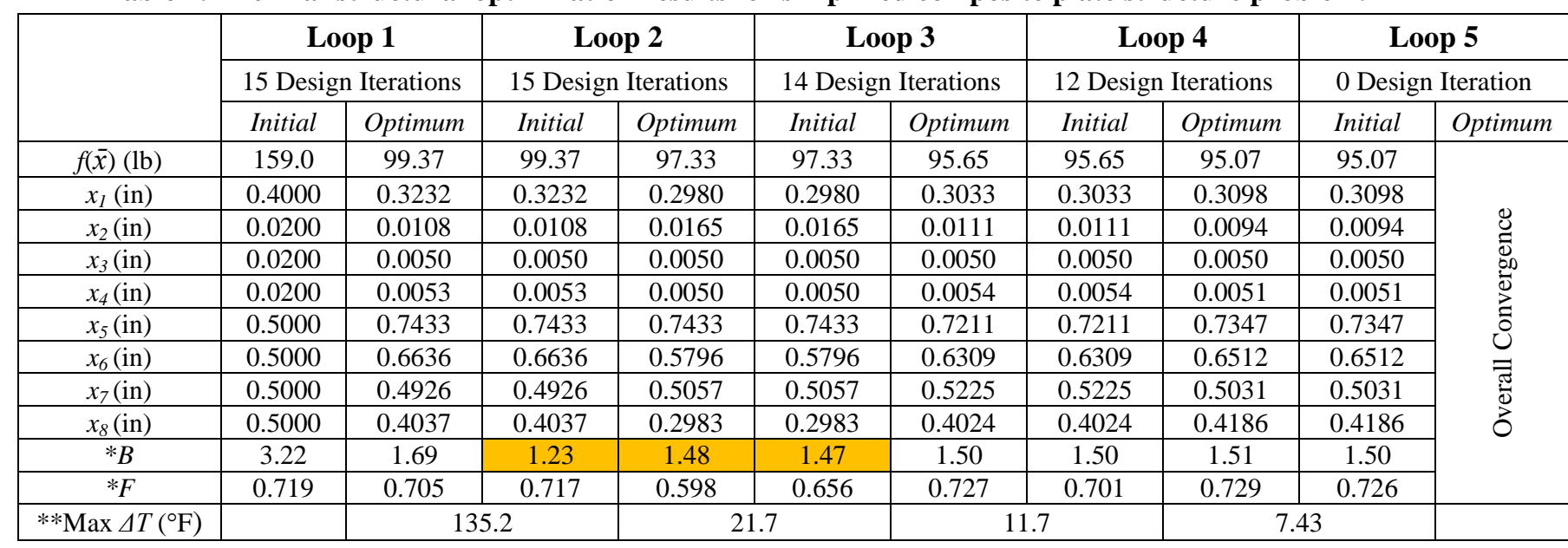

* critical constraint value at any point in time

** maximum absolute change at any grid point at any time between sequential outer loops

violated constraint value 
Throughout optimization, we see the transient temperature fields change based on the design variables change as in the previous example. However, with each outer loop, the difference in the maximum absolute temperature difference between any nodal values at any time between sequential loops continually decreases. In addition, the relative change between critical responses at each loop decreases as well. This demonstrates that the optimization framework is developing optimum solutions for the given load sets, while also converging onto the proper temperature field for the structural optimization in the terminal loop.

The buckling constraint seems to dominate the system response, which is consistent with typical behavior of restrained thin shell structures subjected to elevated temperatures. To best address the buckling constraint, the optimizer varies the thickness of the CMC component, the $0^{\circ}$ plies, and the core thicknesses of the other components. The optimizer sets the thicknesses of the $90^{\circ}$ plies and $45^{\circ}$ plies to the minimum bound because the plies in these orientations do not contribute stiffness in the proper directions to increase the resistance to buckling due to the applied loads. This occurs because the majority of loading exists effectively parallel to the $x-y$ plane (with the exception of a small amount of loading due to thermal expansion in other directions) where the $0^{\circ}$ plies provide the most stiffness. In a more three dimensional, full scale EEWS structure, one would expect that loading would occur in all dimensions such that more stiffness in other directions would be required. However, remaining within the context of this example, assuming that the thickness of a typical graphite ply is approximately 0.005 inches, a practical interpretation of optimization results is as follows. An efficient and manufacturable design for this structure subjected to the prescribed combined thermal-structural loading, where thermal loads are transient in nature consists of a CMC surface of 0.31 inches thick, core thicknesses for components (\#2) to (\#5) of 0.75, 0.65, 0.50, and 0.40 inches respectively, and facesheets with a stacking sequence of $\left[ \pm 45 / 0_{2} / 90\right]_{\mathrm{s}}$ on each sandwich component. Reanalyzing the structure with these parameters yields a possible design that satisfies all constraints with a structural weight of 95.47 pounds.

\section{Conclusion}

In this work, the fundamental thermal-structural response of engine exhaust-washed structures found on embedded engine aircraft has been explored. It was demonstrated that in these structures, various critical thermoelastic responses occurred due to transient thermal loading effects that may not be adequately captured with simple thermal-structural optimization methods based on steady-state thermal results. For example, critical responses occur when different components of the structure experience the largest differences in thermal expansion, which results from significantly different temperatures or different materials in different components. In this system, the instances with largest difference in thermal expansion occur during transient operation rather than once the system has reached steady-state. Therefore, designing a system using only steady-state thermal loading may result in structural failures under actual transient thermal-structural loading conditions, or overly conservative designs, depending on the structural configuration itself and the nature of the transient loading. In addition, a trade space in the sizing of different areas of a conceptual EEWS component was demonstrated that results primarily from the multi-level design dependency of the thermal loading itself. In response, a gradient-based, thermal-structural optimization framework using the commercial codes MD Nastran and GENESIS was developed to aid in the design of these structures at an acceptable level of computational cost. It was demonstrated that by using a sequential loop of structural optimization runs and updating the transient temperature values after each inner optimization convergence, an overall optimum can be reached with relatively few costly transient heat transfer analyses. The process was demonstrated on a simple three bar truss and also a more complex, built up structure made of composite materials. For problems of higher complexity, where structural optimization requires more iterations to converge, the computational cost reduction obtained by this method over including the transient thermal analysis in the optimization loop would likely be more pronounced. In addition, this framework is easily extendable to any combined thermal-structural loading problem where thermal effects are transient in nature. Future work will include expanding the application of thermal-structural optimization to larger scale engine exhaust-washed structures and alternate EEWS configurations in addition to including a constraint filtering scheme to help accommodate the large number of constraints that commonly accompany transient optimization problems. 


\section{Appendix}

Table 3. Mechanical and thermal properties of Ti-6-4 at $700^{\circ} \mathrm{F}$.

\begin{tabular}{|l|l|}
\hline \multicolumn{2}{|c|}{$\mathrm{Ti}^{-6-4}\left(700^{\circ} \mathrm{F}\right)$} \\
\hline Elastic Modulus, $E(\mathrm{Msi})$ & 12.38 \\
\hline Poisson Ratio, $v$ & 0.31 \\
\hline CTE, $\alpha\left(10^{-6} /{ }^{\circ} \mathrm{F}\right)$ & 5.5 \\
\hline Thermal Conductivity, $k\left(\mathrm{Btu} / \mathrm{hr}^{-\mathrm{ft}-}{ }^{\circ} \mathrm{F}\right)$ & 6.7 \\
\hline Specific Heat, $C_{p}\left(\mathrm{Btu} / \mathrm{lb}-{ }^{\circ} \mathrm{F}\right)$ & 0.165 \\
\hline Density, $\rho\left(\mathrm{lb} / \mathrm{in}^{3}\right)$ & 0.160 \\
\hline
\end{tabular}

Table 4. Mechanical and thermal properties of composite materials.

\begin{tabular}{|c|c|}
\hline \multicolumn{2}{|l|}{ Composite Facesheet Material } \\
\hline Longitudinal Modulus, $E_{l}$ (Msi) & 23.5 \\
\hline Transverse Modulus, $E_{2}(\mathrm{Msi})$ & 1.40 \\
\hline Shear Modulus, $G_{I 2}=G_{I 3}(\mathrm{Msi})$ & 0.37 \\
\hline Poisson Ratio, $v_{12}$ & 0.81 \\
\hline Longitudinal CTE, $\alpha_{l}\left(10^{-6} /{ }^{\circ} \mathrm{F}\right)$ & -0.90 \\
\hline Transverse CTE, $\alpha_{2}\left(10^{-6} /{ }^{\circ} \mathrm{F}\right)$ & 16.7 \\
\hline Longitudinal Tension Strength, $X_{t}(\mathrm{ksi})$ & 370.0 \\
\hline Longitudinal Compression Strength, $X_{c}$ (ksi) & 140.0 \\
\hline Transverse Tension Strength, $Y_{t}(\mathrm{ksi})$ & 4.0 \\
\hline Transverse Compression Strength, $Y_{c}(\mathrm{ksi})$ & 35.0 \\
\hline Shear Strength, $S$ (ksi) & 11.2 \\
\hline Longitudinal Conductivity, $k_{I}\left(\mathrm{Btu} / \mathrm{hr}-\mathrm{ft}-{ }^{\circ} \mathrm{F}\right)$ & 7.5 \\
\hline Transverse Conductivity, $k_{2}\left(\mathrm{Btu} / \mathrm{hr}-\mathrm{ft}^{-}{ }^{\circ} \mathrm{F}\right)$ & 0.5 \\
\hline Specific Heat, $C_{p}\left(\mathrm{Btu} / \mathrm{lb}-{ }^{\circ} \mathrm{F}\right)$ & 0.29 \\
\hline Density, $\rho\left(\mathrm{lb} / \mathrm{in}^{3}\right)$ & 0.051 \\
\hline \multicolumn{2}{|c|}{ *Titanium Honeycomb Core Material } \\
\hline$G_{13}(\mathrm{Msi})$ & 0.75 \\
\hline$G_{23}(\mathrm{Msi})$ & 0.775 \\
\hline Thermal Conductivity, $k\left(\mathrm{Btu} / \mathrm{hr}-\mathrm{ft}-{ }^{\circ} \mathrm{F}\right)$ & 1.0 \\
\hline Specific Heat, $C_{p}\left(\mathrm{Btu} / \mathrm{lb}-{ }^{\circ} \mathrm{F}\right)$ & 0.1 \\
\hline Density, $\rho\left(\mathrm{lb} / \mathrm{in}^{3}\right)$ & 0.0055 \\
\hline \multicolumn{2}{|l|}{$* * C M C$ Material } \\
\hline Elastic Modulus, $E_{1}=E_{2}(\mathrm{Msi})$ & 8.0 \\
\hline Shear Modulus, $G_{l 2}(\mathrm{Msi})$ & 2.6 \\
\hline Transverse Shear Modulus, $G_{l 3}=G_{23}(\mathrm{Msi})$ & 1.5 \\
\hline Poisson Ratio, $v_{12}$ & 0.25 \\
\hline CTE, $\alpha_{1}=\alpha_{2}\left(10^{-6} /{ }^{\circ} \mathrm{F}\right)$ & 2.2 \\
\hline Proportional Limit Strength (ksi) & 10.0 \\
\hline Shear Strength, $S$ (ksi) & 3.0 \\
\hline
\end{tabular}

* It is assumed that the honeycomb core can only carry shear loading. It should also be noted that transient thermal effects in honeycomb core are negligible.

** Thermal properties are not required for the CMC material since the part of the model in the second demonstration example made of CMC material has fixed temperature boundary conditions. 


\section{References}

${ }^{1}$ Thornton, E. A., Thermal Structures for Aerospace Applications, AIAA Education Series, AIAA, Reston, VA, 1996.

${ }^{2}$ Gatewood, B. E., Thermal Stresses, McGraw-Hill Book Company, Inc., York, PA, 1957, Chap. 1.

${ }^{3}$ Paterson, J., "Overview of Low Observable Technology and Its Effects on Combat Aircraft Survivability," Journal of Aircraft, Vol. 36, No. 2, 1999, pp. 380-388.

${ }^{4}$ Haney, M. A., "Topology Optimization of Engine Exhaust-Washed Structures," Ph.D. Dissertation, Dept. of Mechanical and Materials Engineering, Wright State University, Dayton, OH, 2005.

${ }^{5}$ Deaton, J. D. and Grandhi, R. V., "Thermal-Structural Analysis of Engine Exhaust-Washed Structures," AIAA Paper 2010-9236, 13th AIAA/ISSMO Multidisciplinary Analysis Optimization Conference, Forth Worth, TX, September 2010.

${ }^{6}$ Haney, M. A. and Grandhi, R. V., "Consequences of Material Addition for a Beam Strip in a Thermal Environment," AIAA Journal, Vol. 47, No. 4, 2009, pp. 1026, 1034.

${ }^{7}$ Bhatia, M., and Livne, E., "Design-Oriented Thermostructural Analysis with External and Internal Radiation, Part 1: Steady State," AIAA Journal, Vol. 46, No. 3, 2008, pp. 578-590.

${ }^{8} \mathrm{Xu}, \mathrm{S}$., and Grandhi, R. V., "Structural Optimization with Thermal and Mechanical Constraints," Journal of Aircraft, Vol. 36, No. 1, 1999, pp. 29-35.

${ }^{9}$ MD Nastran 2010 User's Guide, MSC.Software Corporation, Santa Ana, CA, 2010.

${ }^{10}$ GENESIS Design Manual, Vanderplaats Research \& Development, Inc., Colorado Springs, CO, November 2009.

${ }^{11}$ Haftka, R. T. and Gurdal, Z., Elements of Structural Optimization, 3rd Ed., Kluwer Academic, Norwell, MA, 1992.

${ }^{12}$ MIL-HDBK-5H, Metallic Materials and Elements for Aerospace Vehicle Structures, Department of Defense, Knovel Interactive Edition, 2003, Chap. 5.

${ }^{13}$ Beer, F. P., Johnston, E. R.,. and DeWolf, J. T, Mechanics of Materials, 4th Ed., McGraw-Hill Book Company, Inc., New York, NY, 2006.

${ }^{14}$ CYCOM 5250-4 Prepreg System, Cytec Engineered Materials, Anaheim, CA.

${ }^{15}$ Mechanical Properties: Ti3Al2.5V Honeycomb Core, Benecor, Inc., Witchita, KS.

${ }^{16}$ Gonczy, S. T., and Sikonia, S. T., "NextelTM 312/Silicon Oxycarbide Ceramic Composites," Handbook of Ceramic Composites, edited by N. Bansal, Kluwer Academic Publishers, New York, NY, 2005, pp. 347-373.

${ }^{17}$ Cengel, Y. A., Heat and Mass Transfer: A Practical Approach, 3rd Ed., McGraw-Hill Book Company, New York, NY, 2007.

${ }^{18}$ Hexweb Honeycomb Attributes and Properties, Hexcel Composites, Pleasanton, CA, 1999.

${ }^{19}$ Kassapoglou, C., Design and Analysis of Composite Structures: With Applications to Aerospace Structures, Kluwer Academic, Norwell, MA, 1992.

${ }^{20}$ Gurdal, Z., Haftka, R. T., and Hajela, P., Design and Optimization of Laminated Composite Materials, John Wiley \& Sons, Inc., New York, NY, 1999. 